\title{
AUSTRALIAN JOURNAL OF OUTDOOR EDUCATION
}

Subscription Application/Renewal/Tax Invoice ABN No: 93894394299

I wish to $\square$ subscribe $\square$ renew my subscription to the AJOE

SURNAME:

FIRST NAME:

ORGANISATION:

POSTAL ADDRESS:

STATE:

POSTCODE:

COUNTRY:

EMAIL:

\section{Subscription Rates}

$\square$ Individual Australia / NZ - A $\mathbf{\$ 5 5 . 0 0 ~ ( t w o ~ i s s u e s ) ~ i n c ~ p o s t a g e * ~}$

$\square$ Individual Elsewhere - $\mathbf{A} \mathbf{\$ 7 0 . 0 0}$ (two issues) inc postage

$\square$ Institutional Australia / NZ (Libraries, universities) - A $\$ 95.00$ (two issues) inc postage*

$\square$ Institutional Elsewhere (Libraries, universities) - $\mathbf{A} \$ \mathbf{1 1 0 . 0 0}$ (two issues) inc postage

* Subscription prices listed are inclusive of GST (in Australia)

\section{Payment}

$\square$ Cheque $\square$ Money Order $\square$ Visa $\square$ Mastercard

(Please make cheques / money orders payable, in Australian Dollars, to Outdoor Council of Australia Inc)

Card No: $\quad-\ldots-\ldots \mid-\ldots-1,-\ldots-1,-$

Expiry Date: $\quad-\ldots l+\ldots$ CVV No.

Name on Card:

Signature:

Please choose carefully as you are not entitled to a refund if you simply change your mind.

Please return this form (or a photocopy) along with FULL PAYMENT to:

Outdoor Council of Australia

Or by email to: secretariat@outdoorcouncil.asn.au

c/o Sports House

150 Caxton St, Milton, QLD, 4064

$\mathrm{Ph}+61733699455$

Fax +61733699355 